\title{
Modulating Emission Properties in a Host-Guest Colloidal Quantum Well Superlattice
}

\author{
Junhong Yu, Manoj Sharma, Yimeng Wang, Savas Delikanli, Hamed Dehghanpour Baruj, \\ Ashma Sharma, Hilmi Volkan Demir,* and Cuong Dang*
}

Self-assembly of colloidal nanocrystals into ordered superlattices is a powerful approach to enable novel collective properties which are not available in individual colloids. However, to date, it remains a major challenge to develop a practical route to modulate such collective properties for potential photonic applications. Herein, it is shown that the collective emission properties in colloidal quantum well (CQW) superlattices, including emission color and anisotropy, can be effectively modulated in a binary host-guest architecture. The experimental and theoretical results reveal that excitons of the host (i.e., the undoped $\mathrm{CQWs}$ ) generated by photoexcitation can be controllably harvested by the guest (i.e., the Cu-doped CQWs) for light emission, owing to an exciton hopping assisted exciton trapping process. Such a nano-building block with tunable collective optical properties may enlighten novel colloidal materialbased photonic applications, including optical anti-counterfeiting, next-generation liquid crystal displays, and multifunctional biological markers.

\section{Introduction}

Superlattices constructed by colloidal nanocrystals, which mimic the organization of atoms into a crystal and exhibit unique ensemble properties, have attracted tremendous research interests for the design of novel functional materials. ${ }^{[1-4]}$ Besides the common advantages of the colloidal nanocrystal family to be ingredients to construct superlattices, ${ }^{[5,6]}$ such as wide tunability in geometry/ physical properties and easy-to-handle colloidal states, colloidal quantum wells (CQW) have demonstrated exceptional photophysical properties benefitting from the atomically flat nanostructure and its magic-sized vertical thickness. ${ }^{[7-14]}$ These characteristics, including spectrally pure light emission, ${ }^{[7,8]}$ preferential interactions

J. Yu, M. Sharma, Y. Wang, S. Delikanli, A. Sharma, H. V. Demir, C. Dang LUMINOUS! Centre of Excellence for Semiconductor Lighting and

Displays

School of Electrical and Electronic Engineering

The Photonics Institute (TPI)

Nanyang Technological University

50 Nanyang Avenue, Singapore 639798, Singapore

E-mail:volkan@stanfordalumni.org; hcdang@ntu.edu.sg

M. Sharma, S. Delikanli, H. D. Baruj, H. V. Demir

Department of Electrical and Electronics Engineering and Department of Physics

UNAM Institute of Materials Science and Nanotechnology

Bilkent University

Bilkent, Ankara 06800, Turkey

M. Sharma

ARC Centre of Excellence in Exciton Science

Department of Materials Science and Engineering

Monash University

Clayton Campus, Melbourne, Victoria 3800, Australia

H. V. Demir

School of Physical and Mathematical Sciences

Division of Physics and Applied Physics

Nanyang Technological University

Singapore 639798, Singapore

C. Dang

CINTRA UMI CNRS/NTU/THALES 3288

Research Techno Plaza

50 Nanyang Drive, Border X Block, Level 6, Singapore 637553, Singapore

The ORCID identification number(s) for the author(s) of this article can be found under https://doi.org/10.1002/adom.202101756.

DOI: 10.1002/adom.202101756 between the platelet planes, ${ }^{[9-11]}$ and a highly anisotropic electronic structure, ${ }^{[12-14]}$ have highlighted CQWs as a promising type of building blocks to generate superlattices targeting for photonic applications. superlattices has been made by different groups..$^{[9,10,15-20]}$ Long anisotropic CQW superlattices beyond $10 \mu \mathrm{m}$ have been observed, and the collective properties of the ordered superlattices have been characterized. For example, Murray et al. demonstrated that the self-assembly of CQWs can be directed into ordered superlattices at the liquid-air interface. ${ }^{[15,16]}$ Tisdale et al. ${ }^{[13]}$ and Riedinger et al. ${ }^{[18]}$ revealed that the orientation of CQWs in the superlattice can be controlled by engineering the solvent. Dubertret et al. showed that the self-assembly of CQWS into stacked columnar structures displays polarized emission ${ }^{[9]}$ and a phonon-replica line at low temperature. ${ }^{[10]}$ However, the majority of these efforts on CQW superlattices have focused on structure constructions and understandings of emerging properties. To functionalize the CQW superlattices for photonic applications, it is often desirable to self-assemble the CQWs into a superlattice with programmable collective properties. ${ }^{[21]}$ Unfortunately, this controllability has remained elusive and a daunting challenge to date.

In this work, we propose a binary host-guest superlattice constructed by four monolayers (MLs) CdSe undoped (the host) and $\mathrm{Cu}$-doped CQWs (the guest) to demonstrate the modulation of collective emission properties. Experimental results reveal that the presence of even a few guest ingredients can strongly
So far, considerable progress in the investigations of CQW 
modify the preference of exciton recombination channel via band-edge emission (BE) or copper emission (CE) due to an ultralong exciton hopping range $(>100 \mathrm{~nm})^{[17,20]}$ and an ultrafast exciton hopping rate $(\approx 4 \mathrm{ps}) \cdot{ }^{[11,17,22]}$ By enlarging the vertical dimension of the binary superlattice, more photoexcited excitons are harvested by $\mathrm{Cu}$ dopants and as a result, the greenish and anisotropy photoluminescence (the intrinsic emission properties of $\mathrm{BE})^{[12,13]}$ is gradually changed into the reddish and isotropy photoluminescence (the intrinsic emission properties of CE). ${ }^{[22,23]}$ In addition, we have modeled the excitonic dynamics (including exciton transfer, exciton trapping, and exciton recombination) in the binary superlattice, which can well-reproduce the controllability observed in the experiments. This work, which expands the current understanding of manipulating collective properties in colloidal superlattices, could lead to novel photonic applications such as multimode optical anticounterfeiting, next-generation liquid crystal displays, and multifunctional biological markers.

\section{Results and Discussion}

Undoped CdSe CQWs with a vertical thickness of $1.2 \mathrm{~nm}$ corresponding to four MLs of lattice unit are synthesized according to previously reported procedures..$^{[7,24]}$ After thorough purification, the undoped CQWs covered with oleic acid ligands are dispersed in hexane. As shown in the left panel of Figure 1a, the quantum confinement in the vertical direction of undoped CQWs enables two sharp excitonic transitions, which correspond to the electron/light-hole (maxima at $480 \mathrm{~nm}$ ) and electron/heavy-hole (maxima at $512 \mathrm{~nm}$ ) absorption features (top panel of Figure $1 \mathrm{~b}$ ). Due to the ultrafast relaxation of lightholes to heavy-holes $(<1 \mathrm{ps}),{ }^{[25,26]}$ only greenish BE emission peaked at $514 \mathrm{~nm}$ associated with electron/heavy-hole exciton recombination can be observed in the emission spectrum (the top panel of Figure 1b). Imaging by transmission electron microscopy (TEM) shows that the undoped CQWs have a monodisperse size distribution and a flat square-like shape with an average size of $22 \pm 5 \mathrm{~nm}$ (Figure S1a, Supporting Information), supporting the formation of long-range ordered superlattices.

Copper ions doped into CdSe CQWs can be realized by using both nucleation doping and partial cation exchange. ${ }^{[22,27-30]}$ However, the nucleation doping method generally results in rectangular-shaped CQWs which are difficult for long-range stacking. For this work, we prefer doped and undoped CQWs with similar dimensions to construct the binary superlattice, therefore, we follow our earlier report on partial cation exchange recipes, where we add a controllable amount of copper acetate precursor into a solution of square-shaped undoped four ML core CQWs. ${ }^{[28]}$ It is worth mentioning that under the studied doping range, copper dopants modify neither the geometry of the CQWs (Figure S1b, Supporting Information) nor the essential excitonic absorbance of the CQWs. One can see in the bottom panel of Figure 1b, both electron/heavyhole and electron/light-hole transitions remain unchanged. Here, the role of copper dopants is to introduce emissive midgap states into the CQW host, as shown in the right panel of Figure 1a. After photoexcitation, the heavy-holes can be trapped by the copper sites $\left(\mathrm{Cu}^{1+}+\mathrm{h} \rightarrow \mathrm{Cu}^{2+}\right.$, h denotes the heavy-hole), and then CE (a broad reddish emission band in the bottom panel of Figure $1 b$ ) is activated by the recombination between the captured holes in copper dopants and the electrons in the conduction band of the host CQWs: $\mathrm{Cu}^{2+}+\mathrm{e} \rightarrow \mathrm{Cu}^{1+}+h v(h v$ is the photon energy of CE and e denotes an electron). ${ }^{[23,31,32]}$

We mix the undoped and Cu-doped CQWs dispersed in hexane (the concentration of both is $10 \mathrm{mg} \mathrm{mL}^{-1}$ ) into a total of $\approx 3 \mathrm{~mL}$ volume with an undoped-to-doped molar ratio of 10 . Previously, various groups (e.g., Dubertret, ${ }^{[9,10]}$ Demir, ${ }^{[17,20]}$ and Abécassis ${ }^{[33]}$ ) have demonstrated that adding polar solvents such as ethanol into the CQW dispersions, which are dissolved in nonpolar solvents (such as hexane here), can lower the interaction potentials between the platelet planes of CQWs because the nonpolar capping ligands will force the CQWs to minimize the surface energy to draw away these added polar solvents. ${ }^{[13,20]}$ Therefore, a relatively strong van der Waals attraction between the platelet planes induces the CQWs to stack on top of each other and results in a column-like superlattice.

Here, we adopt the same method to initiate the stacking. To control the degree of stacking, different amounts of ethanol are added to the same pristine mixture. We observe that when a larger amount of ethanol is added into the binary mixture, after stirring for a while, the solution becomes more turbid, suggesting the formation of macroscale particles. ${ }^{\left[{ }^{[9}\right.}$ More interestingly, by tuning the volume of added ethanol, the emission colors of the binary mixture can be modified. Specifically, under UV illumination as shown in Figure 1c, the pristine mixture is greenish since the majority of emitters are undoped CQWs; however, the color of the mixture changes from green to red with more ethanol added, indicating that CE is dominating the emission process. Note that all these four solutions look yellowish under room light. At this point, a legitimate question would be whether the added ethanol changes the intrinsic properties of CQWs. To rule out this possibility, we have investigated again the absorption and emission spectra of the mixtures after adding ethanol. The results in Figure S2 (Supporting Information) reveal that: two excitonic transition features are still located at 480 and $512 \mathrm{~nm}$. Also, the peak position as well as the full-width at half-maximum (FWHM) of both BE and CE display negligible changes. The only changing factor is the intensity ratio between CE and BE. Thus, we can attribute the observed modification to the formation of superlattices.

To better understand the formation of CQW superlattices and characterize the degree of stacking, TEM images of the mixtures with different amounts of ethanol addition are exhibited in Figure 1d. For the pristine mixture (i.e., no ethanol added, see the left TEM panel), all CQWs are in the face-down configuration and no discernible stacking can be observed. When $100 \mu \mathrm{L}$ of ethanol is added into the mixture (see the middle TEM panel), sparsely microwire-like superlattices, in which CQWs are edged-up in a highly aligned columnar order and the principal axis of the superlattice (i.e., the axis along the superlattice) is perpendicular to the platelet plane of CQWs, are detected. As the volume of ethanol increased to $400 \mu \mathrm{L}$ (see the right TEM panel), we notice that almost all CQWs are assembled into the stacking manner to form the superlattices. Even in the direction parallel to the platelet plane of 
(a)

$$
\text { CdSe CQWs }
$$

CB

(d)

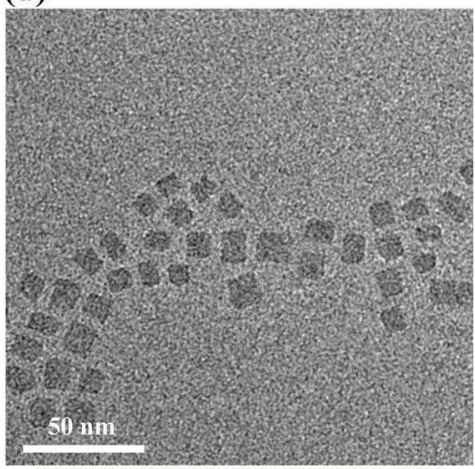

Cu:CdSe CQWs

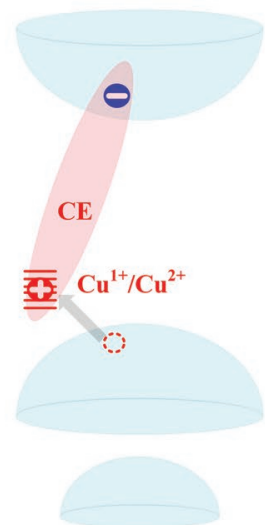

(b)
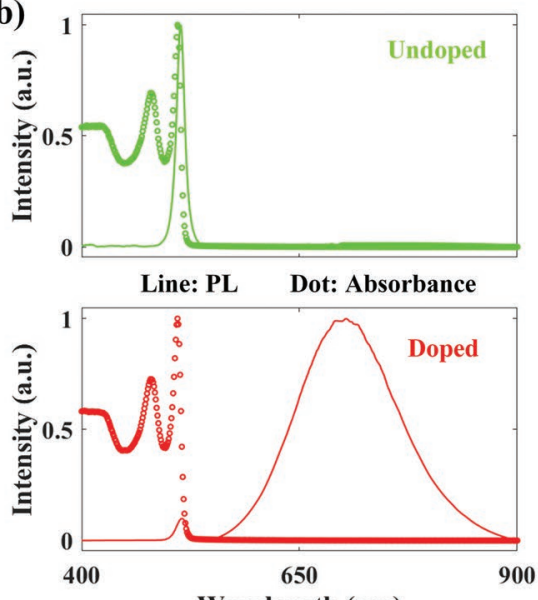

(c)
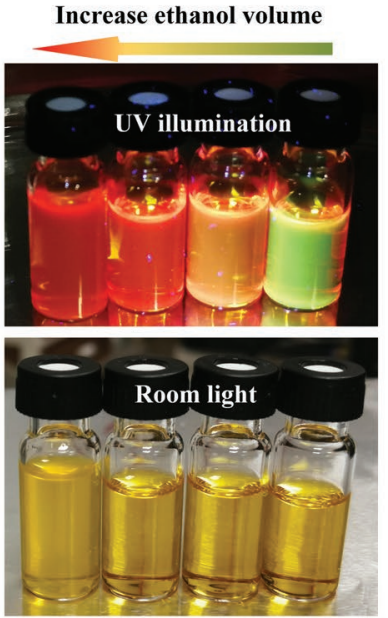

Wavelength (nm)
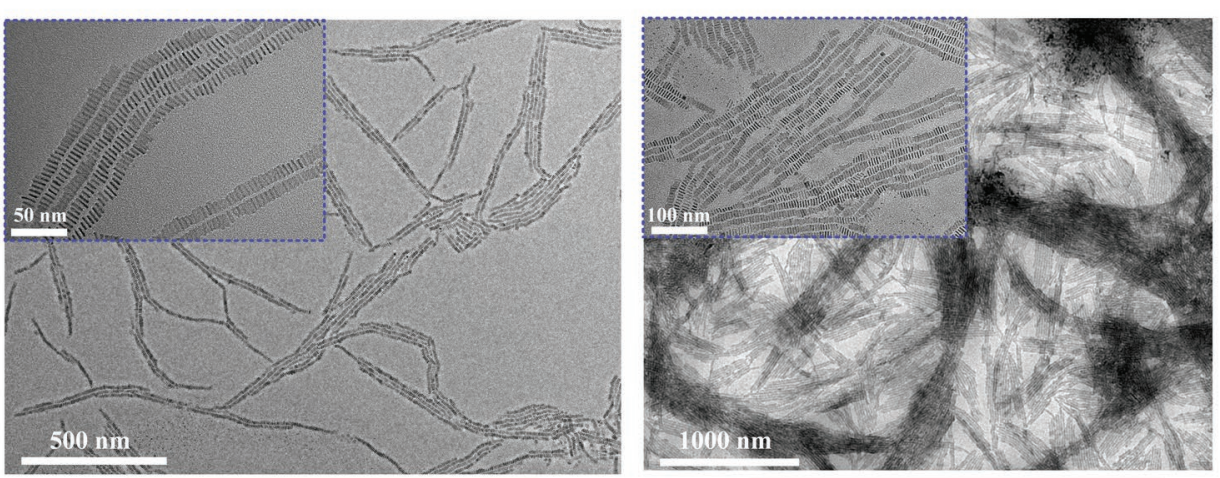

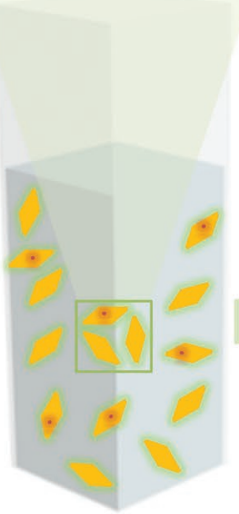

Adding ethanol: $\sim 100 \mu \mathrm{L}$

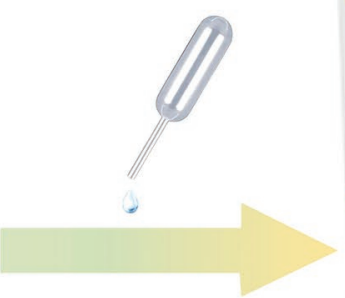

Stirring: $\sim 1$ hour

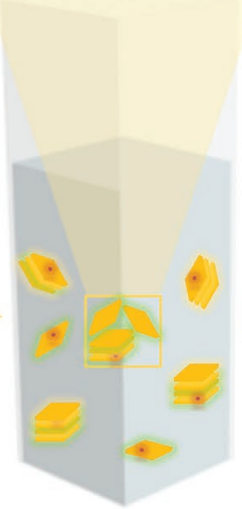

Adding ethanol: $\sim 400 \mu \mathrm{L}$

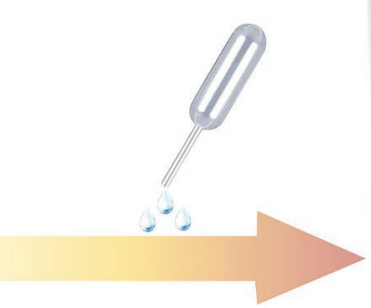

Stirring: 1 hour

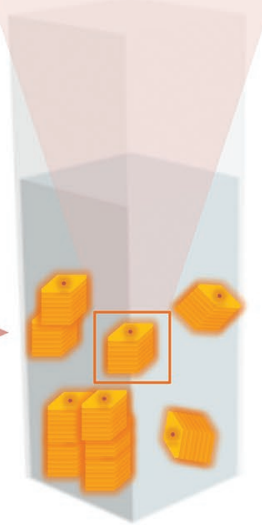

Figure 1. Formation of binary CQW superlattices. a) Schematic illustrations of the excitonic processes in undoped CQWs (left panel) and Cu-doped CQWs (right panel). CB: conduction band, HH: heavy-hole, LH: light-hole. b) Absorption and photoluminescence spectra of undoped CQWs (top panel) and Cu-doped CQWs (bottom panel). c) Color pictures of the binary CQW mixture dispersed in hexane with different amounts of added ethanol under $365 \mathrm{~nm}$ UV lamp (top panel) and under room light (bottom panel). From right to left: the volume of ethanol added into the binary mixture is $0,100,200$, and $400 \mu \mathrm{L}$, respectively. d) Schematic of adding ethanol to obtain CQW superlattices with different stacking degrees and corresponding TEM images of the binary CQWs. As the volume of ethanol is increased gradually, longer and heavier stacking of CQWs into columnar superlattices is observed.

CQWs, more than ten columns of stacked CQWs with a width larger than $100 \mathrm{~nm}$ are standing side by side to form a giant superlattice. It is worth mentioning that small-angle X-ray scat- tering (SAXS) measurements can further confirm the formation of the ordered superlattice and extract the average centerto-center distance between two adjacent CQWs. ${ }^{[9,33-35]}$ 
(a)
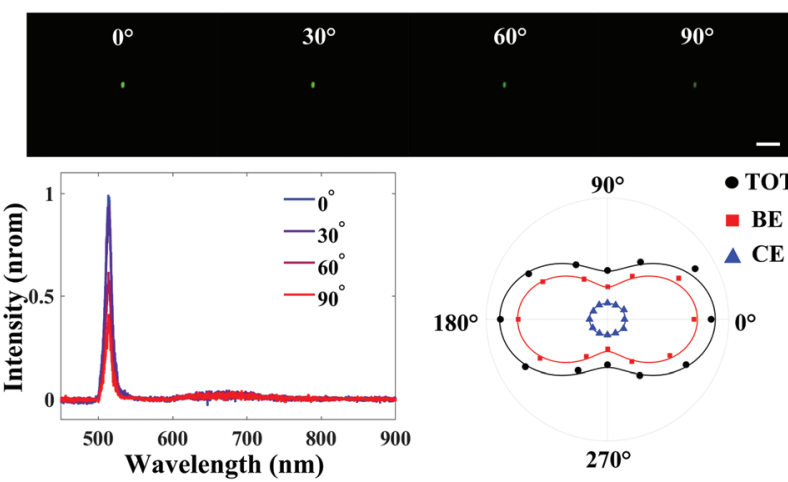

(c)
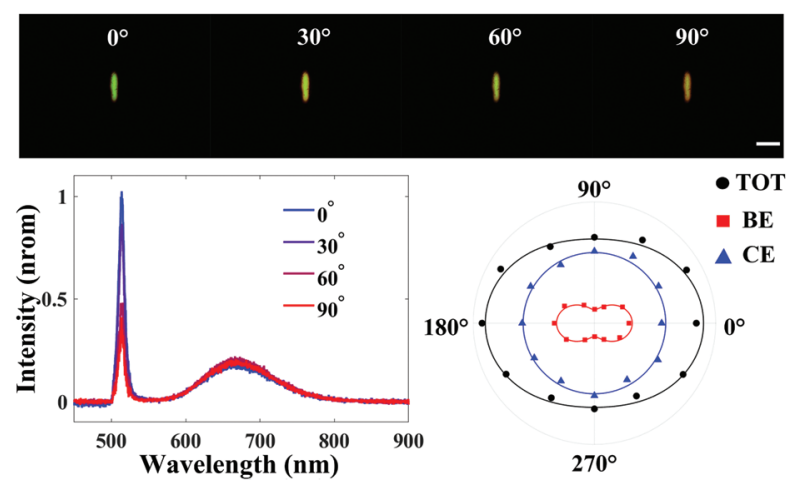

(e)
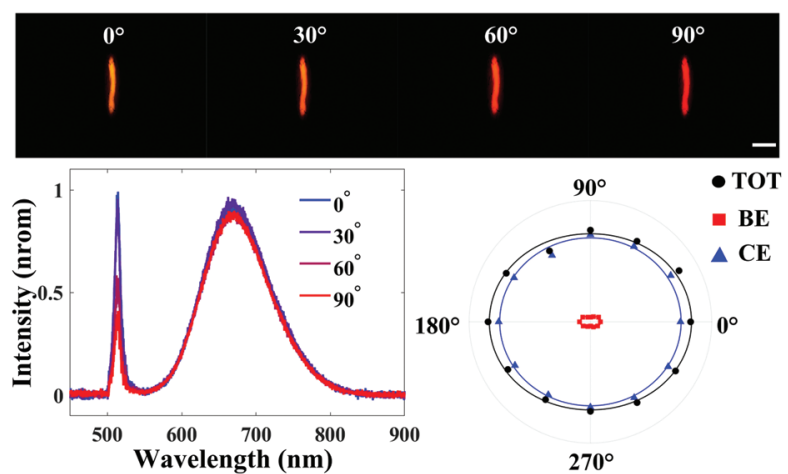

- BE

$\triangle \mathrm{CE}$

$180^{\circ}$

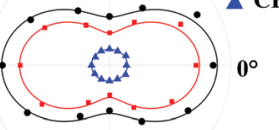

$270^{\circ}$

(b)
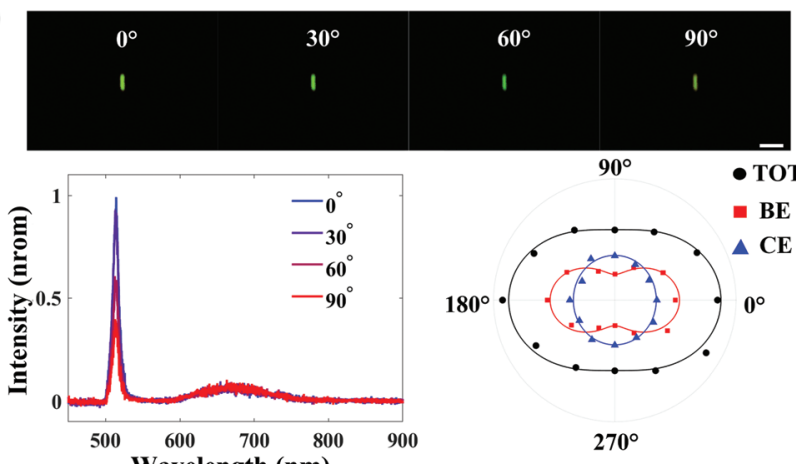

- TOT

(d)
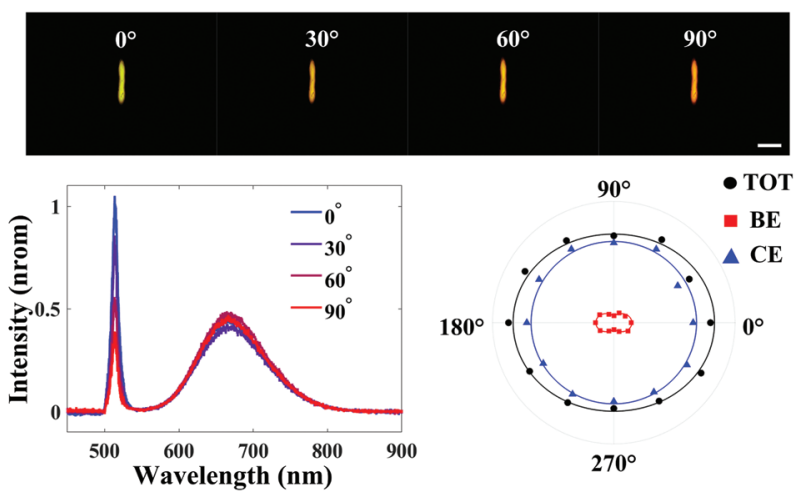

$180^{\circ}$

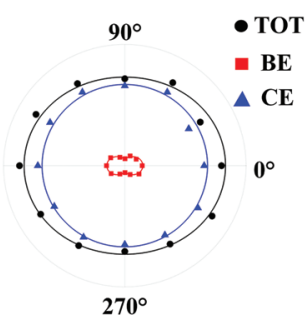

(f)
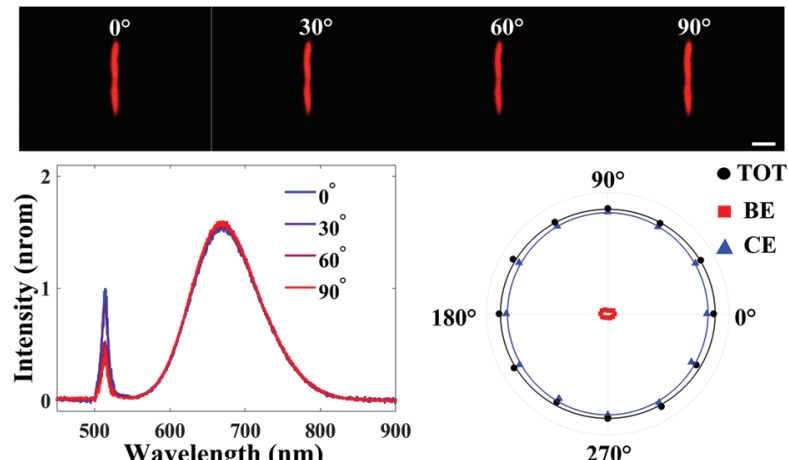

$270^{\circ}$

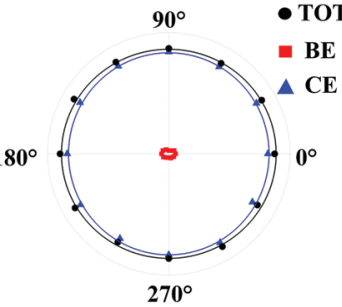

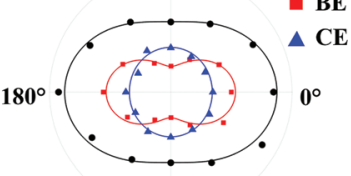

Figure 2. Emission color and anisotropy in the binary CQW superlattices with different stacking lengths. Photoluminescence microscopy images (above), angle-dependent emission spectra (lower-left panel), and angular polarization profiles of emission (lower right panel) of the binary superlattice with a length of a-f) $\approx 1.4, \approx 3.8, \approx 6.7, \approx 9.8, \approx 13.4$, and $\approx 17.2 \mu \mathrm{m}$, respectively. All are excited by a continuous-wave laser (405 nm) with a linear polarization perpendicular to the principal axis of superlattices. The angle between detection polarization and excitation polarization is indicated. In the emission images, the scale bar is $5 \mu \mathrm{m}$. The plotted data in angular profiles are fitted with a sinusoidal function. TOT: normalized integrated intensities of the full (total) emission spectra.

At the aim of providing convincing evidence that the controllability is resulting from the formation of superlattices, it is essential to extract the emission properties of a single superlattice from the ensemble photoluminescence (PL) profile of the dispersion which contains multiple superlattices with different stacking levels. ${ }^{\left[{ }^{[6]}\right]}$ To ensure only an individual superlattice is characterized, after adding $200 \mu \mathrm{L}$ ethanol, a diluted binary mixture dispersion is sparsely deposited onto a glass substrate via drop-casting. Then, each specific superlattice is excited by a linearly-polarized continuous-wave laser $(405 \mathrm{~nm})$ with polarization perpendicular to the principal axis of the superlattice, i.e., parallel to platelet planes. The emission is collected by an angleresolved polarized $\mu$-PL setup (see experimental details in the Experimental Section) to probe the color and anisotropy. ${ }^{[0,37,38]}$
Six superlattices with different stacking lengths have been characterized and the optical images recorded by a CMOS camera allow us to determine the length value. The results of superlattices with lengths of $1.4,3.8,6.7,9.8,13.4$, and $17.2 \mu \mathrm{m}$ are presented in Figure 2. The emission images and spectra of the single superlattice have exhibited consistency with our previous observation in superlattice dispersion (Figure 1c and Figure S2, Supporting Information): the stacking length can modify the intensity ratio between $\mathrm{BE} / \mathrm{CE}$ and a longer superlattice will display the emission color closer to red. Great attention is paid to the angle-resolved polarized emission properties (see the angular polarization profiles in the lower right panel of Figure 2). For all these six superlattices with different stacking lengths, we find that there is a periodical oscillation of the $\mathrm{BE}$ 


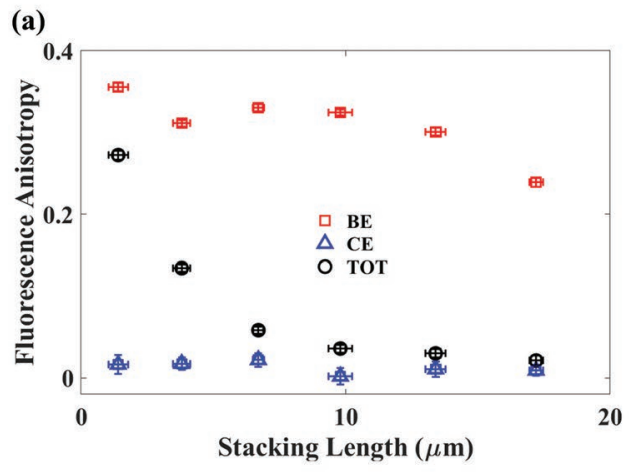

(b)

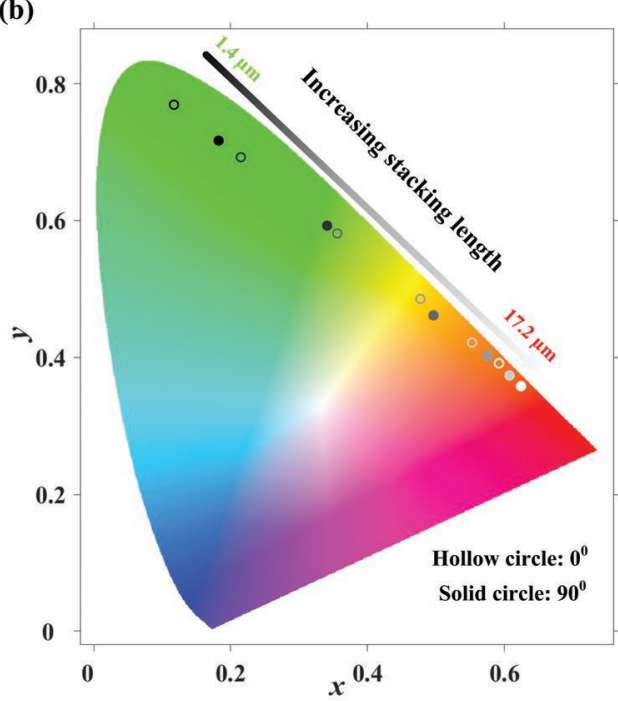

Figure 3. A summary of the achieved controllability. a) Anisotropy as a function of stacking length for BE, CE, and full emission spectra. b) Corresponding color gamut of the emission colors from the superlattice with different stacking lengths when the polarizer is perpendicular to the principal axis of the superlattice $\left(0^{\circ}\right.$, the hollow circles) and the polarizer is parallel to the principal axis of the superlattice $\left(90^{\circ}\right.$, the solid circles).

intensity with respect to polarization angle; and in striking contrast, no periodical variation of CE intensity is observed.

This discrepancy stems from the different preferences of the transition dipole orientation in $\mathrm{BE}$ and $\mathrm{CE}$. It has been widely reported that the electron/heavy-hole exciton transition dipoles in CdSe CQWs are near-perfectly oriented in the platelet plane. ${ }^{[12,13]}$ This pure in-plane dipole orientation can be understood by using the band-edge periodic function $(|u\rangle)$. With spin-orbit interaction (here we define the quantum confinement along $Z$-direction), conduction band electrons have s-like symmetry and $\left|u_{\mathrm{e}}\right\rangle$ can be written as $|1 / 2, \pm 1 / 2\rangle$, while heavy-holes hold $p$-like symmetry and $\left|u_{\text {heavy-hole }}\right\rangle$ takes $\mid 3 / 2$, $\pm 3 / 2\rangle$. We will find that the transition strength (the integral of $\left.\left\langle u_{\mathrm{e}}|\mu| u_{\text {heavy-hole }}\right\rangle\right)$ can only be non-zero for dipoles orientated in the platelet plane (i.e., the $X-Y$ plane). ${ }^{[12]}$ Consequently, in the edge-up configuration (i.e., all platelet planes of CQWs are perpendicular to the substrate and the principal axis in the superlattice), BE (the radiative recombination of electron/heavy-hole exciton) will be the brightest when the principal axis of superlattices is perpendicular to the collection polarizer $\left(I_{0^{\circ}}\right.$, the collection polarization is parallel to the excitation polarization) and be the weakest when the principal axis of superlattices is parallel to the collection polarizer $\left(I_{90^{\circ}}\right.$, the collection polarization is perpendicular to the excitation polarization).

While for $\mathrm{CE}$ (the radiative recombination between the trapped holes and electrons in the host conduction band), transition dipoles do not have any preference and isotropic emission is expected since after the hole is trapped by the copper sites, the Z-projection of total angular momentum is not fixed at $\pm 3 / 2$ anymore and the in-plane conservation has been broken. Similar interpretations can be found in the light-holes $(|u\rangle=|3 / 2, \pm 1 / 2\rangle)$ and spinoff-holes $(|u\rangle=|1 / 2,1 / 2\rangle)$, in which the dipole orientation is projected in all space directions. ${ }^{[12]}$ We can also calculate the anisotropy value $(R)$ of BE and CE using: ${ }^{[19,37-39]}\left(I_{0^{\circ}}-I_{90^{\circ}}\right) /\left(I_{0^{\circ}}+2 \times{ }_{90^{\circ}}\right)$ to quantize the contrast. As shown in Figure 3a, CE yielded an anisotropy value of nearly zero, indicating no net anisotropy (i.e., isotropy). While for BE, with a short stacking length, the anisotropy value is above 0.3 , which is comparable to the results in colloidal nanorods; ${ }^{[19,39]}$ with longer stacking length, the anisotropy value decreases to $\approx 0.2$ possibly because CQWs are harder to maintain pure edge-up configurations in such a long chain (over $15 \mu \mathrm{m}$ ). Benefitting from the huge anisotropy value contrast in $\mathrm{BE} /$ $\mathrm{CE}$, modification of full-spectrum anisotropy will be concomitantly achieved when the intensity ratio between $\mathrm{CE}$ and $\mathrm{BE}$ is tuned by the stacking length. In Figure 3a, we can see that in the short-stacked superlattice, the full-spectrum is highly anisotropic since $\mathrm{BE}$ is dominating the emission spectra, and by the increase of stacking length, the total emission of the superlattice becomes almost isotropic. Moreover, colour coordinates of the two polarizations of emission spectra for six superlattice lengths are plotted in the CIE 1931 chromaticity diagram (Figure 3b), which shows impressive color controllability ranging from green to red. It is worth mentioning that if proper filters are adopted, such as polarization filters, one can further enhance the color purity by utilizing the contrast anisotropy values of $\mathrm{BE}$ and $\mathrm{CE}$.

After demonstrating the capability of the stacking length to modify the collective emission color and anisotropy in superlattices, the remaining task is to clarify the underpinning physics that enable the controllability. Based on our knowledge of CdSe CQWs from previous works, the most reasonable hypothesis is that the exciton sinking in the Cu-doped CQWs (associated with $\mathrm{CE}$ ) is boosted by efficient and long-range exciton transfer in superlattices. Let us start with the superlattice constructed by pure undoped CdSe CQWs. Due to the physically close dipoledipole interaction (various groups have reported a spatial period of $<5 \mathrm{~nm}$ in CdSe CQW stacking ${ }^{[11,17,20,22]}$ ), pure in-plane dipole orientation and a large self-overlap between the emission/ absorption spectra, photoexcited band-edge excitons could transfer back and forth among the adjacent CQWs via Förster resonance energy transfer (FRET), which is a nonradiative 
(a)

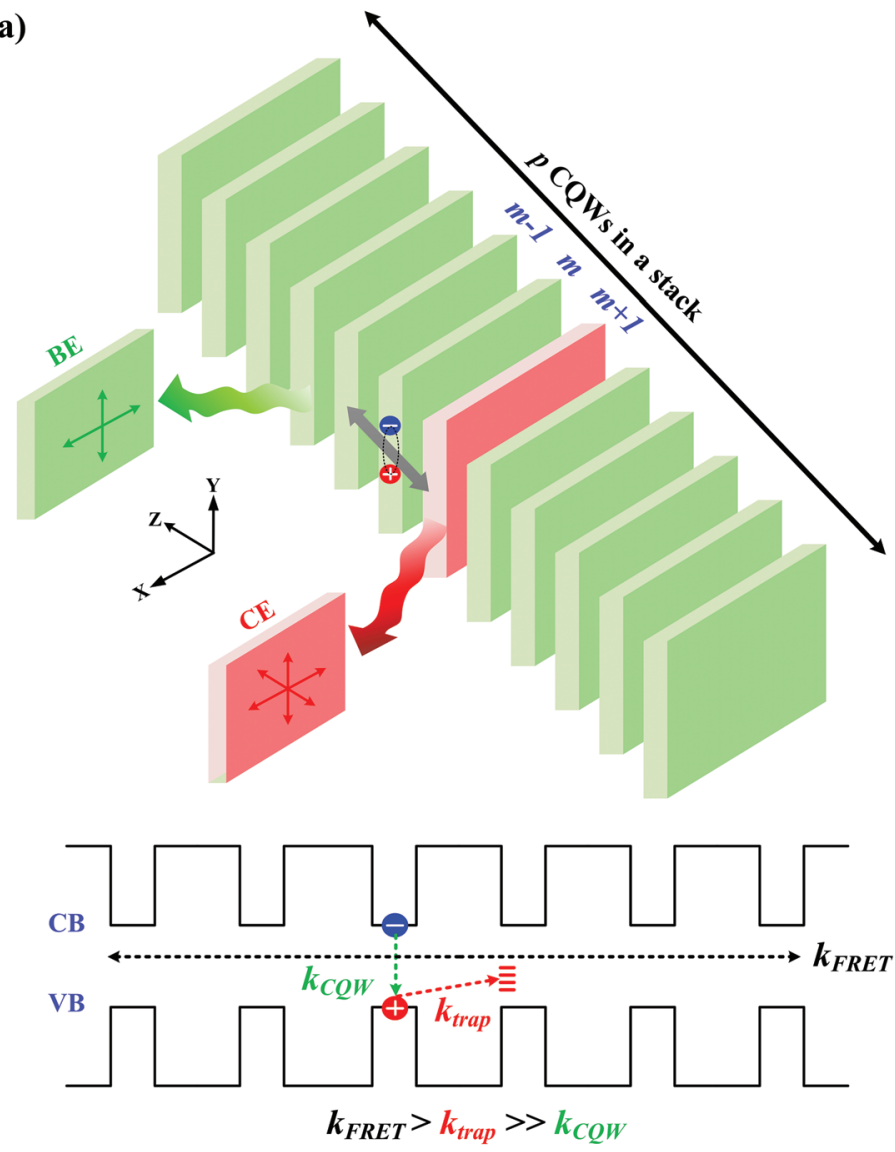

(b)

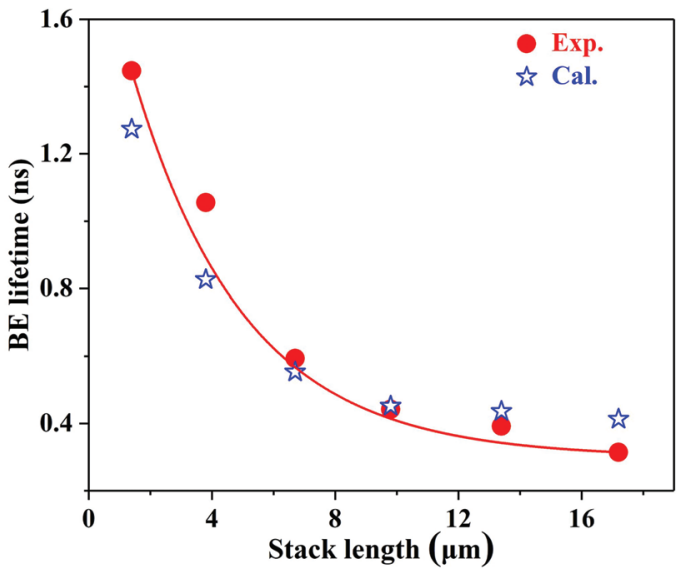

(c)

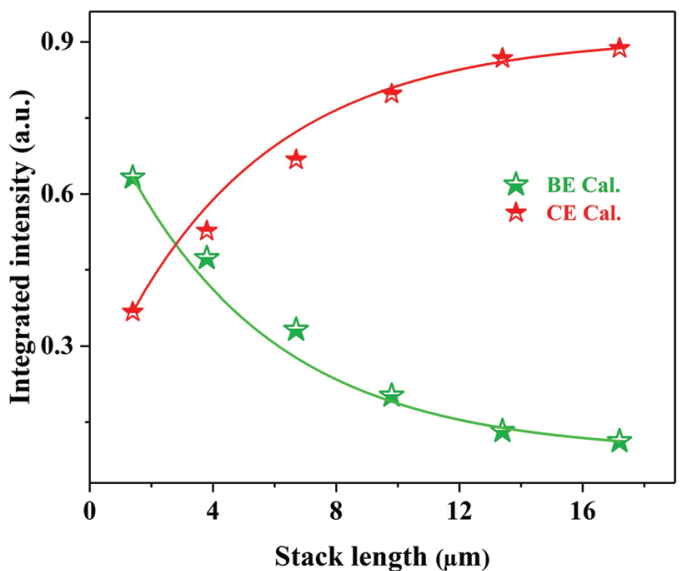

Figure 4. Controlling mechanism in the binary CQW superlattice. a) Schematic illustration of FRET assisted exciton trapping process in the binary superlattice. Green shaded plates denote undoped CQWs in which BE is greenish and anisotropic due to the in-plane dipole orientation. While red shaded plates represent Cu-doped CQWs in which CE is reddish and isotropic due to the lack of dipole orientation preference. Among all these exciton dynamics, exciton transfer via FRET is the fastest: $k_{F R E T}$ is $(\approx 3 \mathrm{ps})^{-1}$ and exciton trapping by copper sites has the lifetime of several tens of picosecond $\left(k_{\text {trap }}\right)$, the slowest process is band-edge exciton recombination $\left(k_{\mathrm{CQ} w}\right)$, which is in the nanosecond range. b) Correlating the simulated BE lifetime (the hollow stars) with the experimental value (the solid circles) to determine the fraction of the Cu-doped CQW population in the superlattice. The red line is a guide to the eye. c) The calculated BE/CE intensity with various stacking lengths. The agreeable trend exhibits very good support for our mechanism interpretation. The two solid lines are the guides to the eye.

exciton transfer process via near-field dipolar coupling. Rowland et al. in 2015[15] and Guzelturk et al. in 2014 ${ }^{[20]}$ reported that the exciton transfer in CdSe CQW stacking can be as fast as $\approx 3$ ps with a near-unit efficiency and outpace the Auger recombination. Considering the lifetime of band-edge recombination is around $3 \mathrm{~ns}$, excitons can hop about a thousand times in the column-like assembly before annihilation.

Now we insert the Cu-doped CQWs which behave as the exciton trappers into the stacks, as shown in the schematic illustration in Figure 4a, taking advantage of the multiple transfer loops of band-edge excitons, the copper sites with a hole trapping lifetime of several tens picosecond ${ }^{[23,31]}$ could capture tremendous photo-excited excitons generated in both doped and undoped CQWs. The scenario in our study is an emissive analogue to previous observations that demonstrated emission of chromophores or nanocrystals is quenched in the assembly due to non-emissive trapping assisted by homoFRET. ${ }^{[17,20,40]}$ As the stacking length is getting longer (i.e., the number of Cu-doped CQWs in the stacking is larger), more of the band-edge excitons will be affected by the exciton trapping process boosted by exciton transfer. As a result, the excitation energy is more likely to be released through the CE channel. We have checked the BE dynamics (probed at $514 \mathrm{~nm}$ ) of nonstacking CQWs and stacking CQWs with different lengths to support our argument (Figure S3, Supporting Information). The CQWs have an $\approx 2.42$-ns average emission lifetime before stacking is induced, which is in accordance with other four ML CdSe CQWs reports. ${ }^{[13,17,25]}$ As the superlattice is formed, the average $\mathrm{BE}$ lifetime can decrease by an order of magnitude to $\approx 320$ ps for the longest stacking. This indicates that the new band-edge exciton consuming channel (i.e., exciton trapping) becomes much stronger with the increase of stacking length.

To further confirm our inference and develop a deeper understanding, we perform a kinetic simulation based on a set of rate equations ${ }^{[17,20,41,42]}$ to reproduce the observed controllability (see the simulation details in Note S1, Supporting Information). For simplicity, we assume the fraction $(f)$ of the $\mathrm{Cu}$ doped CQW population within the whole CQW population in all superlattices is fixed (because the superlattice constructed from the uniformly mixed dispersion, the stacking with a con- 
siderable amount of CQWs should contain a similar population percentage of doped ingredient) and there is no nonradiative loss in the superlattice. To extract the $f$ value in our experiments, we have swept this parameter from 0.01 to 0.20 to correlate the simulated BE dynamics with the measured BE decays in Figure S3 (Supporting Information) and found acceptable accordance when $f=0.13$ (as shown in Figure 4b). Then we can determine the photoexcited energy-releasing via $\mathrm{BE}$ and $\mathrm{CE}$ channels: the $\mathrm{BE}$ energy is calculated by integrating the simulated $\mathrm{BE}$ dynamics in a relatively long-time window while the CE energy is obtained by subtracting $\mathrm{BE}$ photons from the photoexcited photons. The stacking-lengthdependent BE/CE intensity from the kinetic model is shown in Figure 4c. Note that we do not expect to achieve good quantitative matching between experiment and simulation results due to the simplification of the model and the random nature of the stacking process. Nevertheless, our simulation results exhibit a good trend in agreement with experimental results: a longer stacking length results in more photoexcited energy released via the CE channel and the collective emission of the superlattice becomes more reddish and isotropic. This again supports our hypothesis that FRET assisted exciton trapping is the dominating mechanism for the collective emission property control.

\section{Summary}

In summary, we have proposed and demonstrated that the emission properties in a host-guest superlattice consisting of undoped and $\mathrm{Cu}$-doped CQWs are highly controllable through a solvent engineer approach. The physical origin of the controllability lies in the exciton hopping boosted exciton trapping process. As the vertical dimension increases, the ultra-efficient homo-FRET leads to more excitons sinking into the Cu-doped CQWs. Therefore, the copper emission, which is reddish and isotropic, gradually overwhelms the band-edge emission, which is greenish and anisotropic. Moreover, a kinetic model has been developed, which strongly agrees with the observed modulation in our experiments. Given the simplicity and flexibility in tuning collective properties of the superlattice, we anticipate that our work could be, in the future, exploited for practical photonic applications.

\section{Experimental Section}

Steady-State Optical Properties of Cu-Doped and Undoped Dispersion: Absorption spectra of CQWs in hexane were measured by a UV-vis spectrophotometer (Shimadzu, UV-1800). PL spectra of CQWs in hexane were recorded using a spectrofluorophotometer (Shimadzu, RF-5301PC, excitation wavelength: $355 \mathrm{~nm}$ ).

Characterization of Emission Color and Anisotropy in Binary CQW Superlattice: The excitation source is a $405-\mathrm{nm}$ continuous-wave laser (Cobolt 06-MLD), and the laser spot was focused into the superlattice by a microscope objective (ZEISS, NA $=0.65$ ). The linear excitation polarization was achieved by using the combination of a linear polarizer and a $\lambda / 2$ wave-plate. The glass substrate hosting superlattices was mounted in a motion stage, which can move along $X$ - and $Y$-direction to bring different superlattices with various stacking lengths into the field of view, and also can rotate to make sure the principal axis of the superlattice is perpendicular to the excitation polarization. The emission was collected by the same objective in the reflection geometry and the excitation laser was filtered out by a long-pass (LP) filter. Then the emission was passed through the collection polarizer and a 50/50 beam splitter, one path was recorded by a colorful CMOS camera and another path was into the fiber-coupled ANDOR spectrometer (monochromator: ANDOR Shamrock 303i, CCD: ANDOR iDus 401). By rotating the collection polarizer in front of the beam splitter, the angle-dependent emission color and anisotropy can be probed.

Time-Resolved Photoluminescence in Binary CQW Superlattice: The time-resolved PL spectroscopy was performed with a Becker \& Hickl DCS 120 confocal scanning FLIM system with the laser pulse at $375 \mathrm{~nm}$ with the repetition rate of $20 \mathrm{MHz}$. For all the time-resolved PL measurements, the collection time is $180 \mathrm{~s}$. Third-order exponential decay models were used to fit the emission dynamics in Figure S6 (Supporting Information) and the instrument response function (IRF) was deconvoluted during the fitting.

\section{Supporting Information}

Supporting Information is available from the Wiley Online Library or from the author.

\section{Acknowledgements}

The authors would like to acknowledge the financial support from Singapore National Research Foundation under the program of NRFNRFI2016-08, the Competitive Research Program NRF-CRP14-2014-03, and the Singapore Ministry of Education AcRF Tier-1 grant (MOE2019-T1-002-087). H.V.D. is also grateful to acknowledge additional financial support from the TUBA.

\section{Conflict of Interest}

The authors declare no conflict of interest.

\section{Author Contributions}

J.Y. and M.S. contributed equally to this work. C.D. and H.V.D. supervised and contributed to all aspects of the research. J.Y. initiated the idea and discussed experiments with M.S. and C.D. in detail. J.Y. wrote the manuscript with the inputs from all authors. With inputs from H.V.D., M.S. designed the doped CQWs and A.S. performed the material synthesis. J.Y. and M.S. performed the experiments to control the degree of stacking. S.D. and H.D.B. conducted the TEM measurement to characterize the stacking. J.Y. and Y.W. conducted the steady-state and dynamic spectroscopy measurements. J.Y. and C.D. performed the kinetic model simulation. All authors analyzed the data, discussed the results, and commented on the manuscript.

\section{Data Availability Statement}

The data that support the findings of this study are available from the corresponding author upon reasonable request.

\section{Keywords}

colloidal quantum wells, colloidal superlattices, Cu-doping, modulating emission properties, self-assembled 
Received: August 22, 202

Revised: November 13, 2021

Published online:

[1] Z. Nie, A. Petukhova, E. Kumacheva, Nat. Nanotechnol. 2010, 5, 15.

[2] E. V. Shevchenko, D. V. Talapin, N. A. Kotov, S. O'Brien, C. B. Murray, Nature 2016, 439, 55.

[3] D. V. Talapin, E. V. Shevchenko, M. I. Bodnarchuk, X. Ye, J. hen, C. B. Murray, Nature 2009, 461, 964.

[4] T. Wang, J. Zhuang, J. Lynch, O. Chen, Z. Wang, X. Wang, D. LaMontagne, H. Wu, Z. Wang, Y. C. Cao, Science 2012, 338, 358.

[5] Y. Nagaoka, H. Zhu, D. Eggert, O. Chen, Science 2018, 362, 1396.

[6] Y. Nagaoka, R. Tan, R. Li, H. Zhu, D. Eggert, Y. A. Wu, Y. Liu, Z. Wang, O. Chen, Nature 2018, 561, 378.

[7] S. Ithurria, M. D. Tessier, B. Mahler, R. P. S. M. Lobo, B. Dubertret, A. L. Efros, Nat. Mater. 2011, 10, 936.

[8] J. Yu, C. Dang, Cell Rep. Phys. Sci. 2021, 2, 100308.

[9] B. Abécassis, M. D. Tessier, P. Davidson, B. Dubertret, Nano Lett. 2014, 14, 710.

[10] M. D. Tessier, L. Biadala, C. Bouet, S. Ithurria, B. Abécassis, B. Dubertret, ACS Nano 2013, 7, 3332.

[11] C. E. Rowland, I. Fedin, H. Zhang, S. K. Gray, A. Govorov, D. V. Talapin, R. D. Schaller, Nat. Mater. 2015, 14, 484.

[12] R. Scott, J. Heckmann, A. V. Prudnikau, A. Antanovich, A. Mikhailov, N. Owschimikow, M. Artemyev, J. I. Climente, U. Woggon, N. B. Grosse, A. W. Achtstein, Nat. Nanotechnol. 2017, 12, 1155.

[13] Y. Gao, M. C. Weidman, W. A. Tisdale, Nano Lett. 2017, 17, 3837.

[14] J. Yu, S. Hou, M. Sharma, Y. M. Tobing, Z. Song, S. Delikanli, C. Hettiarachchi, D. Zhang, W. Fan, M. D. Birowosuto, H. Wang, H. V. Demir, C. Dang, Matter 2020, 2, 1550.

[15] T. Paik, D.-K. Ko, T. R. Gordon, V. Doan-Nguyen, C. B. Murray, ACS Nano 2011, 5, 8322

[16] X. Ye, J. Chen, M. Engel, J. A. Millan, W. Li, L. Qi, G. Xing, J. E. Collins, C. R. Kagan, J. Li, S. C. Glotzer, C. B. Murray, Nat. Chem. 2013, 5, 466

[17] O. Erdem, M. Olutas, B. Guzelturk, Y. Kelestemur, H. V. Demir J. Phys. Chem. Lett. 2016, 7, 548.

[18] R. Momper, H. Zhang, S. Chen, H. Halim, E. Johannes, S. Yordanov, D. Braga, B. Blülle, D. Doblas, T. Kraus, M. Bonn, H. I. Wang, A. Riedinger, Nano Lett. 2020, 20, 4102.

[19] P. D. Cunningham, J. B. Souza, I. Fedin, C. X. She, B. Lee, D. V. Talapin, ACS Nano 2016, 10, 5769

[20] B. Guzelturk, O. Erdem, M. Olutas, Y. Kelestemur, H. V. Demir, ACS Nano 2014, 8, 12524.

[21] M. A. Boles, M. Engel, D. V. Talapin, Chem. Rev. 2016, 116, 11220.
[22] J. Yu, M. Sharma, S. Delikanli, M. D. Birowosuto, H. V. Demir, C. Dang, J. Phys. Chem. Lett. 2019, 10, 5193.

[23] H. D. Nelson, X. Li, D. R. Gamelin, J. Phys. Chem. C 2016, 120, 5714.

[24] S. Ithurria, B. Dubertret, J. Am. Chem. Soc. 2008, 130, 16504.

[25] M. Pelton, S. Ithurria, R. D. Schaller, D. S. Dolzhnikov, D. V. Talapin, Nano Lett. 2012, 12, 6158.

[26] L. Biadala, F. Liu, M. D. Tessier, D. R. Yakovlev, B. Dubertret M. Bayer, Nano Lett. 2014, 14, 1134.

[27] M. Sharma, K. Gungor, A. Yeltik, M. Olutas, B. Guzelturk, Y. Kelestemur, T. Erdem, S. Delikanli, J. R. McBride, H. V. Demir, Adv. Mater. 2017, 29, 1700821.

[28] M. Sharma, M. Olutas, A. Yeltik, Y. Kelestemur, A. Sharma, S. Delikanli, B. Guzelturk, K. Gungor, J. R. McBride, H. V. Demir, Chem. Mater. 2018, 30, 3265.

[29] J. Yu, M. Sharma, M. Li, S. Delikanli, A. Sharma, M. Taimoor, Y. Altintas, J. R. McBride, T. Kusserow, T. C. Sum, H. V. Demir C. Dang, Laser Photonics Rev. 2021, 15, 2100034

[30] J. Yu, M. Sharma, M. Li, P. L. Hernandez-Martinez, S. Delikanli, A. Sharma, Y. Altintas, C. Hettiarachchi, T. C. Sum, H. V. Demir, C. Dang Sustained biexciton emission in colloidal quantum wells assisted by dopant-host interaction. arXiv 2019, 1905.11571.

[31] K. E. Hughes, K. H. Hartstein, D. R. Gamelin, ACS Nano 2018, 12, 718

[32] V. Pinchetti, Q. Di, M. Lorenzon, A. Camellini, M. Fasoli, M. Zavelani-Rossi, F. Meinardi, J. Zhang, S. A. Crooker, S. Brovelli, Nat. Nanotechnol. 2018, 13, 1145

[33] S. Jana, M. de Frutos, P. Davidson, B. Abécassis, Sci. Adv. 2017, 3 , e1701483.

[34] S. Jana, P. Davidson, B. Abecassis, Angew. Chem. 2016, 128, 9517.

[35] S. Jana, T. N. T. Phan, C. Bouet, M. D. Tessier, P. Davidson, B. Dubertret, B. Abecassis, Langmuir 2015, 31, 10532.

[36] J. Cui, A. P. Beyler, I. Coropceanu, L. Cleary, T. R. Avila, Y. Chen, J. M. Cordero, S. L. Heathcote, D. K. Harris, O. Chen, J. Cao, M. G. Bawendi, Nano Lett. 2016, 16, 289.

[37] E. Cassette, B. Mahler, J.-M. Guigner, G. Patriarche, B. Dubertret, T. Pons, ACS Nano 2012, 6, 6741.

[38] M.-J. Sun, Y. Liu, Z. Wei, Y. S. Zhao, Y.-W. Zhong, J. Yao, J. Am. Chem. Soc. 2019, 141, 6157.

[39] Y. Li, H. Huang, Y. Xiong, A. F. Richter, S. V. Kershaw, J. Feldmann, A. L. Rogach, ACS Nano 2019, 13, 8237.

[40] B. Guzelturk, M. Olutas, S. Delikanli, Y. Kelestemur, O. Erden, H. V. Demir, Nanoscale 2015, 7, 2545.

[41] J. Yu, M. Sharma, A. Sharma, S. Delikanli, H. V. Demir, C. Dang, Light: Sci. Appl. 2020, 9, 27.

[42] J. Yu, S. Shendre, W.-K. Koh, B. Liu, M. Li, S. Hou, C. Hettiarachchi, S. Delikanli, P. Hernandez-Martínez, M. D. Birowosuto, H. Wang, T. Sum, H. V. Demir, C. Dang, Sci. Adv. 2019, 5, eaav3140. 